\title{
OPEN Homogeneity of Arabian Peninsula dromedary camel populations with signals of geographic distinction based on whole genome sequence data
}

\begin{abstract}
Hussain Bahbahani ${ }^{1 \bowtie}$ \& Faisal Almathen ${ }^{2,3}$
Dromedary camels in the Arabian Peninsula distribute along different geographical and ecological locations, e.g. desert, mountains and coasts. Here, we are aiming to explore the whole genome sequence data of ten dromedary populations from the Arabian Peninsula to assess their genetic structure, admixture levels, diversity and similarity indices. Upon including reference dromedary and Bactrian camel populations from Iran and Kazakhstan, we characterise inter-species and geographic genetic distinction between the dromedary and the Bactrian camels. Individual-based alpha genetic diversity profiles are found to be generally higher in Bactrian camels than dromedary populations, with the exception of five autosomes (NC_044525.1, NC_044534.1, NC_044540.1, NC_044542.1, NC_044544.1) at diversity orders $(q \geq 2)$. The Arabian Peninsula camels are generally homogenous, with a small degree of genetic distinction correlating with three geographic groups: North, Central and West; Southwest; and Southeast of the Arabian Peninsula. No significant variation in diversity or similarity indices are observed among the different Arabian Peninsula dromedary populations. This study contributes to our understanding of the genetic diversity of Arabian Peninsula dromedary camels. It will help conserve the genetic stock of this species and support the design of breeding programmes for genetic improvement of favorable traits.
\end{abstract}

The domestic one-humped Camelus dromedarius (dromedary camel) and two-humped Camelus bactrianus (Bactrian camel), in addition to the wild two-humped camelus ferus, form the old world Camelini tribe of the Camelidae family. The ancestors of this tribe, which diverged from the new world Lamini tribe about 16.3 million years ago (Mya), reached Eurasia via the Bering land bridge approximately 6.5-7.5 Mya. After the divergence of the one-humped camels from the two-humped animals, around 4.4 Mya, the Bactrian camels were domesticated from their wild ancestors about 5000-6000 years ago, most probably in eastern Asia. This was followed by the domestication of the dromedary camels in the southeastern region of the Arabian Peninsula around 3000-4000 years ago ${ }^{1}$.

Dromedary and Bactrian camels show wide geographical distribution due to their historical use in longdistance trading and transportation. Dromedary camels are predominantly found in the desert and semi-arid regions of Africa, Arabian Peninsula and southwest Asia, while Bactrian camels are mainly distributed throughout eastern and central Asia ${ }^{2}$. In several countries, including for instance Iran, India, Turkey and Kazakhstan, both domestic camel types can be found, and anthropogenic hybridization between them can result in animals with high robustness and endurance-commonly used along long-distance trade routes ${ }^{1}$.

In the Arabian Peninsula, dromedary camels are classified according to various criteria, for example coat color, ecological location and their productivity, however there is no established breeding system informed by genetic analysis. Regarding coat color, camels with dark brown to black coat colors are known as Magaheem, while camels with white coats are known as Wodeh. Sofor and Shual camels have a brown coat color, with Sofor camels mainly characterized as being dark brown to black at the top of their neck, shoulder, hump and tail ${ }^{3}$. All of the above

${ }^{1}$ Department of Biological Sciences, Faculty of Science, Kuwait University, Al-Shidadya, Kuwait. ${ }^{2}$ Department of Public Health, College of Veterinary Medicine, King Faisal University, 400, Al-Hasa, Kingdom of Saudi Arabia. ${ }^{3}$ Camel Research Center, King Faisal University, 400, Al-Hasa 31982, Kingdom of Saudi Arabia. ${ }^{\square}$ email: hussain.bahbahani@ku.edu.kw 
mentioned camel populations are used for milk and meat production, are hence classified as production camels, and can be found in the north and central area of the Arabian Peninsula. Camels bred specifically for competitive racing are hence known as racing camels, and are mainly from either Oman in the southeast of the Arabian Peninsula, or the north of the Arabian Peninsula where a recognized population known as al-Hurra is popular ${ }^{4,5}$.

Dromedary camels from the west, e.g. Hadana and Sahlia, and southwest of Arabian Peninsula, e.g. Awadi and Awarik, face different ecological conditions than those from the north and center of the Arabian Peninsula. In contrast to the wide desert covering the north and center of the Arabian Peninsula, Hadana and Awadi camels are populating higher elevations, on the tops mountains, and are hence known as Hill or Mountain camels. By contrast, Sahlia and Awarik camels are mainly found near the Red Sea coast of Saudi Arabia and are known as Beach camels ${ }^{5}$.

Assessing the genetic diversity and structure of dromedary camels is the first milestone towards establishing a standard genetically informative breeding programme. Until recently this field of research has largely been confined to the analysis of autosomal microsatellite markers and partial mitochondrial DNA (mtDNA) sequences ${ }^{6-8}$, while more recent efforts by Bahbahani et al. ${ }^{9}$ and Ming et al. ${ }^{10}$ employed genotype-by-sequence (GBS) and whole genome sequence data, respectively. A fundamental challenge in assessing the genetic diversity of dromedary populations concerns accessibility to camel samples representing the different populations, and defining informative statistics with which to evaluate their diversity. Several diversity indices exist that measure species diversity at ecological levels, which can also be used to evaluate the genetic diversity of livestock species ${ }^{11,12}$. Shannon's entropy, Simpson's index and Renyi's entropy are examples of such indices, which translated mathematically into number of equivalents called Hill numbers, behave in a way that can be used to express diversity ${ }^{11}$. All of these indices decompose into two components; alpha and beta diversities, which describe different diversity angles. Alpha diversity measures the average diversity of a single ecological community, while beta diversity measures the relative change in species composition between ecological communities ${ }^{11}$. An important parameter that differs between the Hill numbers of these two diversity components is the diversity order (q), which determines the sensitivity of the measure to the frequency of the species ${ }^{13}$. At $q=0$, the species frequency, or abundance, is not counted. While, at $\mathrm{q}=1$, the species are weighed in proportion to their frequency. At $\mathrm{q}>1$, the Hill number becomes sensitive to the most frequent species $^{11,13}$.

Recently, Ma et al. ${ }^{12}$ applied these ecological diversity measures to quantify SNP diversity at the level of the individual. These proposed alpha and beta diversity measures are able to account for the uneven, non-random, distribution of SNPs along chromosomes. Ma et al. ${ }^{12}$ proposed four Hill-number-based similarity measures to compare SNP similarity between populations. These measures investigate SNPs similarities at different levels: local SNP overlap $\left(\mathrm{Cq}_{\mathrm{N}}\right)$ that quantifies the proportion of shared SNPs among individuals; regional SNP overlap $(\mathrm{UqN})$ that looks at the proportion of shared SNPs in the pooled population; SNP homogeneity $\left(\mathrm{Sq}_{\mathrm{N}}\right)$ that measures SNP evenness between populations; and SNP turnover complement $\left(\mathrm{Vq}_{\mathrm{N}}\right)$ that represents the relative rate of SNP turnover per individual.

In this study, we investigate if the different Arabian Peninsula dromedary camel populations can be genetically discriminated based on their geographical distribution using whole genome sequence data. Individual-level diversity and similarity indices are assessed in these populations and compared to reference dromedary and Bactrian camels.

\section{Results}

Summary statistics of mapped sequence reads. The mean depth of coverage of the mapped sequence reads among the dromedary samples ranged from 13.7 to $32.16 \times$, with a mean of $25 \times$, while among Bactrian camels it ranged from 5.96 to $23.02 \times$ with a mean of $13.3 \times$ (Supplementary Table S1). On average, $99.82 \%$ of the dromedary sequence reads and $99.61 \%$ of Bactrian sequence reads mapped to the dromedary reference genome (CamDro3), with $94.4 \%$ and $94.7 \%$ of mapped reads remaining properly paired in dromedaries and Bactrians, respectively. The average proportions of reference genome covered by the mapped reads were $93.25 \%$ in dromedaries and $90.84 \%$ in Bactrians (Supplementary Table S1).

Genetic structure and relatedness. Principle component analyses (PCA) analysis of all camels (dataset 1) reveals clear differentiation between dromedary and Bactrian populations along the first principle component (PC1), explaining $37.8 \%$ of the total variation. Whilst, the second principle component (PC2), accounting for $3.2 \%$ of the total variation, separates camels from Iran and the Arabian Peninsula populations (Fig. 1a).

A PCA focused only on the Arabian Peninsula dromedary camels (dataset 2) reveals structure broadly consistent with the geographic sampling. Dromedaries sampled from the west, southwest and southeast of the Peninsula (Hadana, Sahlia, Awarik, Awadi and Omani) are separated from those sampled from the north and center of the Arabian Peninsula populations (Hurra, Sofor, Shual, Wodeh, Magaheem) along PC1, explaining 6.7\% of the total variation. PC2, which accounts for $6.2 \%$ of the total variation, shows some separation of the Hurra samples and Awadi camels from the other populations, in addition to an Awarik and a Shual samples (Fig. 1b).

The mean relatedness $\Phi$ between the Arabian Peninsula dromedary samples is $0.108 \pm 0.094$ indicating them to be second-degree relatives (Supplementary Table S2). A single first-degree relationship was identified between a Hurra and a Shual, which are separated from their corresponding populations as revealed by the PCA plot on Arabian Peninsula dromedary camels (Fig. 1b).

Admixture analyses were performed to further investigate the genetic structure of both dromedary and Bactrian camels. The optimal number of clusters identified in dataset 1 (Fig. 2a) was $K=2$ (Supplementary Table S3a). This $K$ value differentiates between the dromedary and Bactrian camels. At this $K$ value the dromedary camels from Iran share genetic background with Arabian Peninsula dromedaries, with one sample shows substantial Bactrian genetic introgression with ancestry proportion value $\sim 0.43$. A signal of distinct genetic background 
a

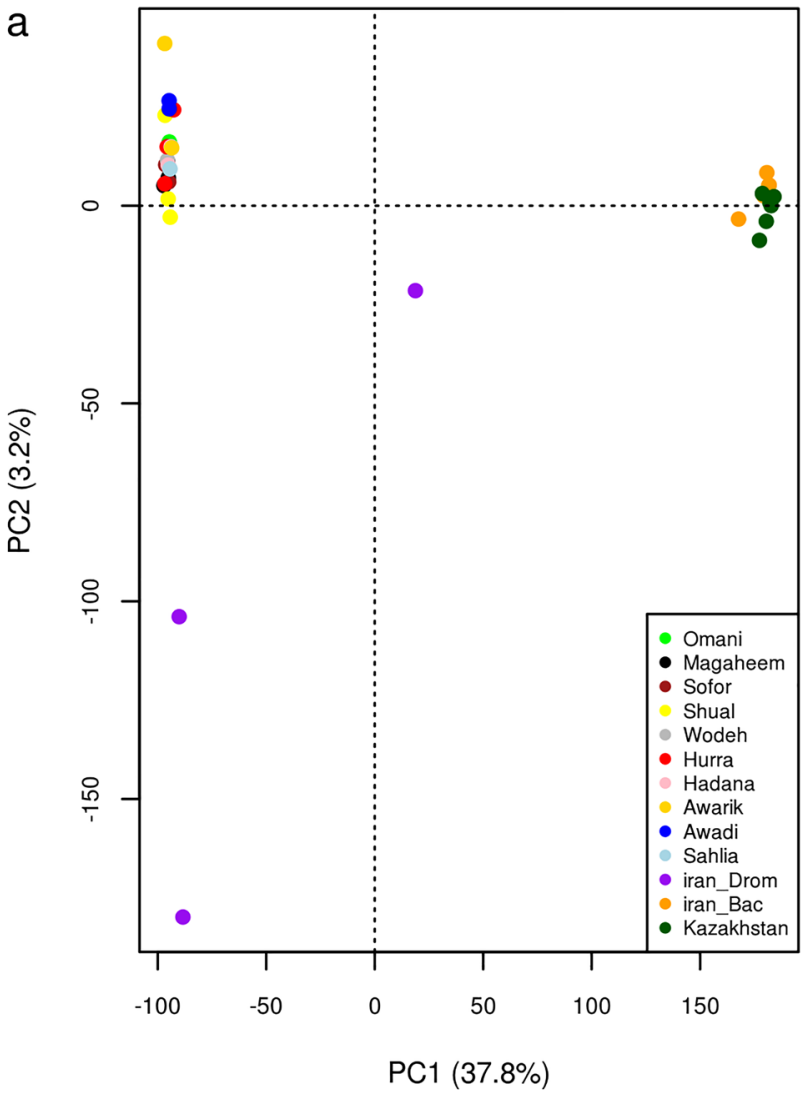

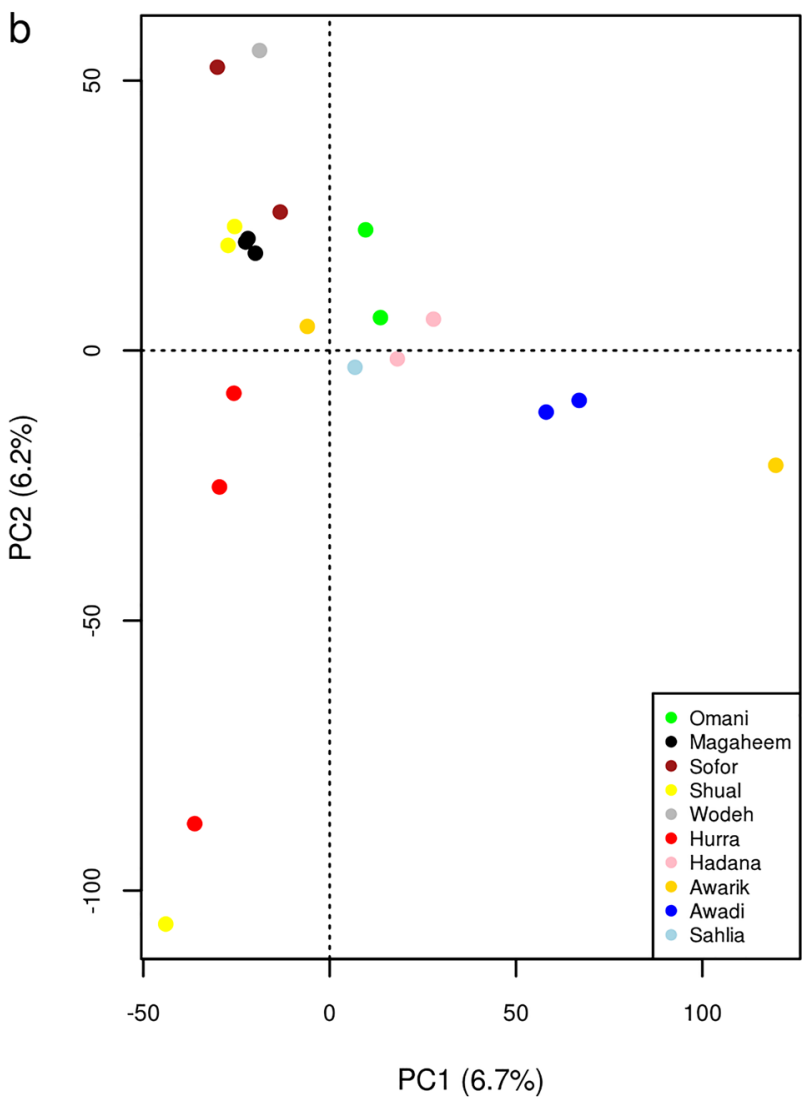

Figure 1. Principle component analysis (PCA) plots on (a) dromedary and Bactrian camels and (b) dromedary camels from the Arabian Peninsula.

corresponding to dromedaries from Iran has emerged at $K=3$, which is more clearly revealed at $K=5$. At $K=4$, a separate genetic ancestry is revealed for the Bactrian camels from Iran, with the exception of two samples carrying a substantial genetic background of Kazakhstan ancestry (ancestry proportion >0.8) (Supplementary Fig. S1).

Admixture analysis of dataset 2 (Fig. 2b) reveals the optimal number of clusters to be $K=1$ (Supplementary Table S3b). At $K=2$, distinct genetic background emerges for dromedary camels from the southwest of the Arabian Peninsula; Awadi and Awarik camels, with the exception of a single Awarik camel mainly carrying a proportion of genetic ancestry $(\sim 0.92)$ not related to the southwest of Arabian Peninsula. A substantial proportion of the southwest genetic ancestry is observed in camel populations from the west; Hadana $(\sim 0.21)$ and Sahili $(\sim 0.16)$, and southeast; Omani $(\sim 0.15)$, of the Arabian Peninsula. At $K=3$, a genetic background specific to Omani camels from the southeast of the Arabian Peninsula is revealed (Fig. $2 \mathrm{~b}$ ). Beyond $K=3$ varying levels of admixture across most camels are observed (Supplementary Fig. S2).

Individual-level SNP diversity. Autosomal SNP diversity profiles were generated for each camel to enable alpha diversity to be calculated at the individual-level. Alpha diversity was observed to be significantly higher (Mann-Whitney $U$ test $P$-value $<0.05$ ) in Bactrian camel populations than in dromedary camels at all diversity orders, except q=0 (Fig. 3; Supplementary Table S4). No statistically significant differences in the average autosomal alpha diversity are observed between dromedary populations, or between Bactrian Populations (Supplementary Table S4). On average, we observe higher alpha diversities in Bactrian camel populations on all but five autosomes, with these exceptions showing significantly higher diversity values in dromedaries at diversity orders q $\geq 2$ (Table 1; Supplementary Table S5; Supplementary Fig. S3). Among the Arabian Peninsula dromedaries, no significant difference in alpha diversity is observed among the different populations for any of the diversity orders tested (Supplementary Fig. S4; Supplementary Table S6).

To further characterise population differentiation, beta diversity and similarity indices were calculated within and between populations for diversity orders $q=0$ to 4 . Analysing dataset 1 , we compared: (1) dromedary from the Arabian Peninsula; (2) dromedary from Iran; (3) Bactrian camels from Iran; and (4) Bactrian camels from Khazakhstan. At diversity orders $\mathrm{q} \geq 1$, We observed higher beta diversity and lower similarity indices when comparing between species (mean beta $=1.34, \mathrm{Cq}=0.49, \mathrm{Uq}=0.70, \mathrm{Sq}=0.52, \mathrm{Vq}=0.66$ ), than within species (mean beta $=1.09, \mathrm{Cq}=0.84, \mathrm{Uq}=0.92, \mathrm{Sq}=0.86, \mathrm{Vq}=0.91$ ) (Figs. 4 and 5; Supplementary Table S7). A comparison of dromedary from Iran to the Bactrian camels returned lower beta diversity and higher similarity indices (mean beta $=1.29, \mathrm{Cq}=0.55, \mathrm{Uq}=0.73, \mathrm{Sq}=0.58, \mathrm{Vq}=0.71)$ than when comparing dromedary from the Arabian Peninsula to Bactrian camels (mean beta $=1.38, \mathrm{Cq}=0.43, \mathrm{Uq}=0.65, \mathrm{Sq}=0.46, \mathrm{Vq}=0.62$ ) at diversity orders $\mathrm{q} \geq 1$ (Figs. 4 and 5; Supplementary Table S7). Analysis of only the Arabian Peninsula dromedary camels (dataset 2) 
a

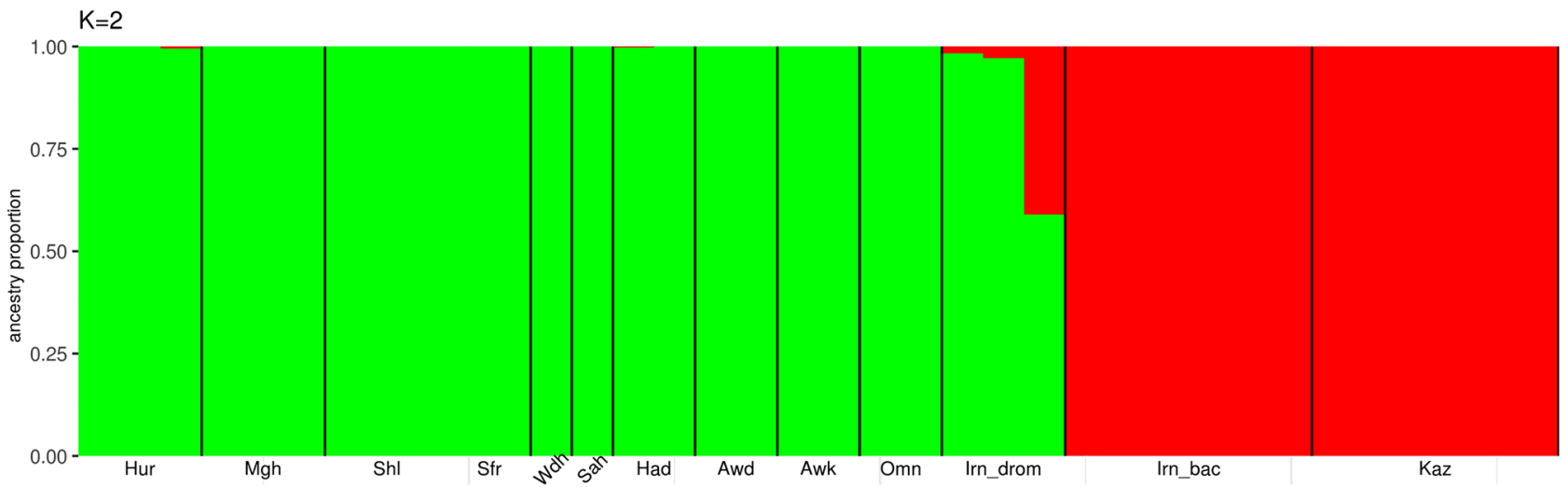

b
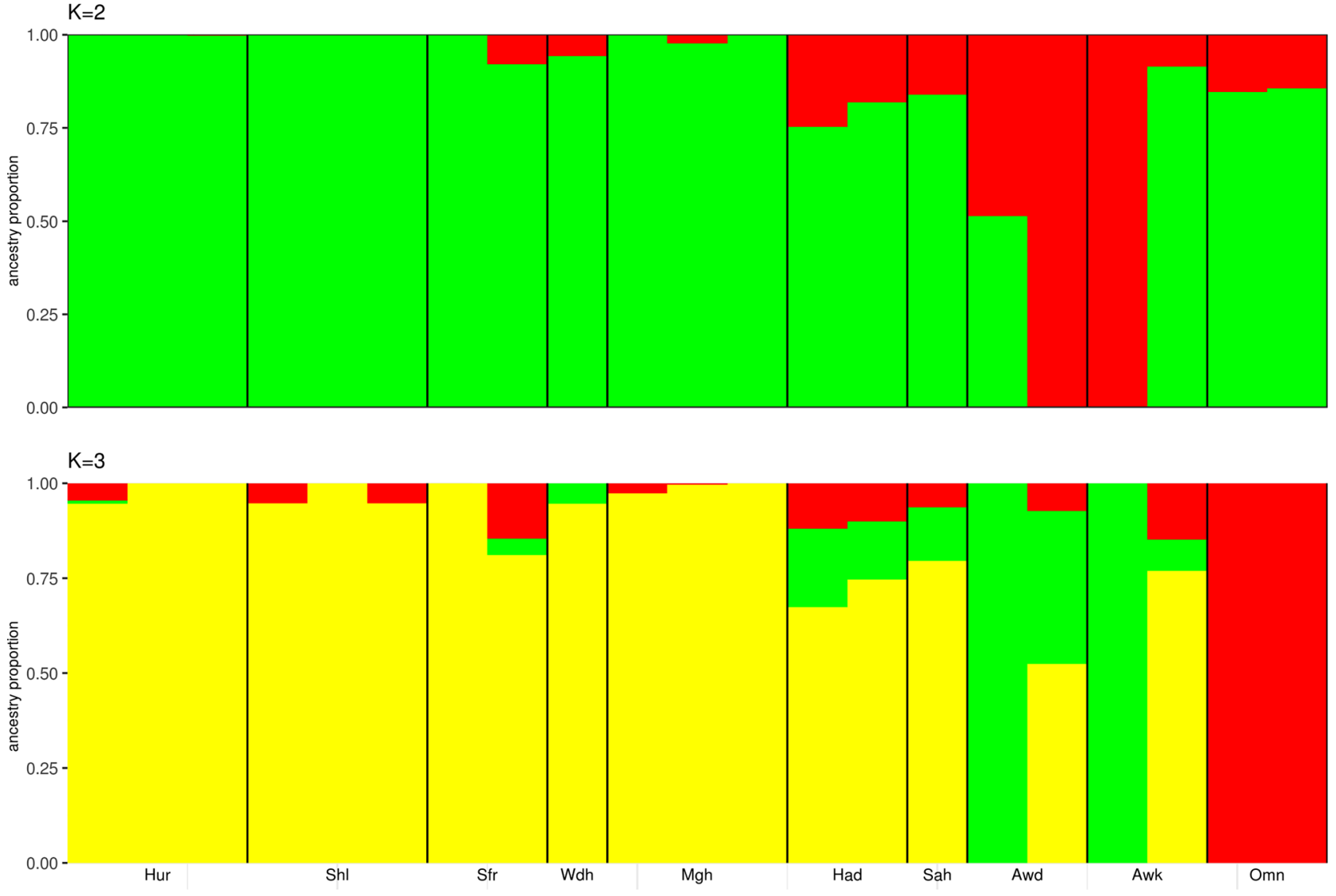

Figure 2. Admixture analysis plots of (a) $K=2$ on the dromedary and Bactrian camels (dataset 1 ) and (b) $K=2$ and 3 on the dromedary camels from the Arabian Peninsula (dataset 2). Hur Hurra, Mgh Magaheem, Shl Shual, Sfr Sofor, Wdh Wodeh, Sah Sahlia, Had Hadana, Awd Awadi, Awk Awarik, Omn Omani, Irn_drom Dromedary from Iran, Irn_Bac Bactrian from Iran, Kaz Bactrian from Kazakhastan.

reveals no significant variation in beta diversity and similarity indices between the different populations at all diversity orders tested (Supplementary Table S8).

\section{Discussion}

The inter-species genetic distinction observed between the dromedary and Bactrian camels revealed by both the PCA and admixture analysis likely results from more than $4 \mathrm{M}$ years of divergence between these two species ${ }^{1}$. Evidence of hybridization between these species can be observed in countries where they coexist, such as Iran, Kazakhstan and Turkey. This genetic hybridization, which explains the introgressed Bactrian genetic ancestry into a dromedary sample from Iran at $K=2$ of the dataset 1 admixture analysis, has previously been detected by Ming et al. ${ }^{10}$, and it is associated with breeding camels that combine the robustness and endurance of both 


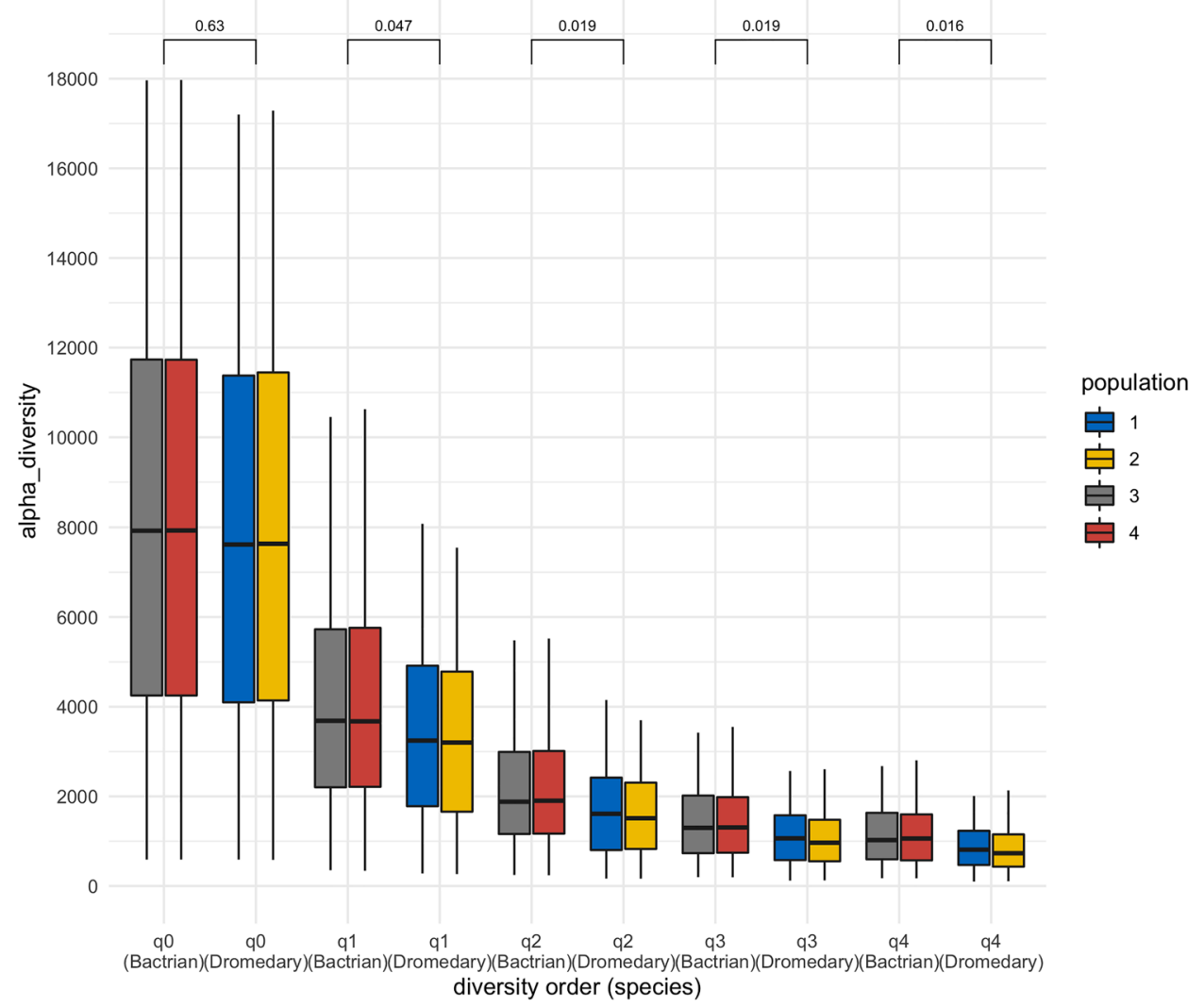

Figure 3. Mean autosomal alpha diversity at the diversity orders $q=0$ to 4 . Populations: (1) Arabian Peninsula dromedary camels; (2) dromedary camels from Iran; (3) Bactrian camels from Iran; and (4) Bactrian camels from Kazakhstan. Mann-Whitney $U$ test $P$-values for pairwise comparisons of dromedary ( 1 and 2$)$ against Bactrian (3 and 4) are reported.

\begin{tabular}{|l|l|l|l|l|l|}
\hline Autosome & q0_P_value & q1_P_value & q2_P_value & q3_P_value & q4_P_value \\
\hline NC_044525.1 & 1.0000 & 0.9996 & 0.0285 & 0.0003 & 0.0000 \\
\hline NC_044534.1 & 1.0000 & 0.1200 & 0.0000 & 0.0000 & 0.0000 \\
\hline NC_044540.1 & 1.0000 & 1.0000 & 1.0000 & 0.9776 & 0.0025 \\
\hline NC_044542.1 & 1.0000 & 1.0000 & 0.2661 & 0.0006 & 0.0001 \\
\hline NC_044544.1 & 1.0000 & 0.9998 & 0.1888 & 0.0191 & 0.0148 \\
\hline
\end{tabular}

Table 1. Mann-Whitney $U$ test $P$-values for autosomes showing significantly higher alpha diversities in dromedaries than Bactrian camels at different diversity orders $(\mathrm{q})$.

species to withstand long-distance journeys ${ }^{1}$. The nar (male) and nar-maya (female) camels in Kazakhstan, and tulu camel in Turkey are examples of such cross-breeding as reviewed by Dioli ${ }^{14}$.

This level of inter-species genetic distinction is also evidenced in the individual-level alpha and beta diversity profiles and similarity indices. However, as the SNPs used in these analyses were derived from aligning sequencing reads to the dromedary camel reference genome assembly (CamDro3), it is inevitable that a greater number of SNPs would be identified in the more divergent species, resulting in higher alpha diversity values in Bactrian camels. Nonetheless, we observed higher alpha diversity profiles in dromedary camels than Bactrians on five autosomes, which may indicate that diversity in the genes on these autosomes may be of functional significance to dromedary camels. This is an interesting observation that warrants further investigation, for example using signatures of selection and gene-wise association analyses with potentially relevant traits.

We observed a degree of differentiation by PCA, admixture and diversity analyses, between the dromedaries from the Arabian Peninsula and those from Iran, which may further be clarified upon including more dromedary samples from Iran. This differentiation is likely resulted from geographical separation by the sea preventing gene flow. Such geographical-wise genetic distinction, has also been observed among African dromedary populations, from Algeria and Egypt ${ }^{15}$, and between African and Arabian Peninsula dromedaries ${ }^{9}$. 
$q=1$

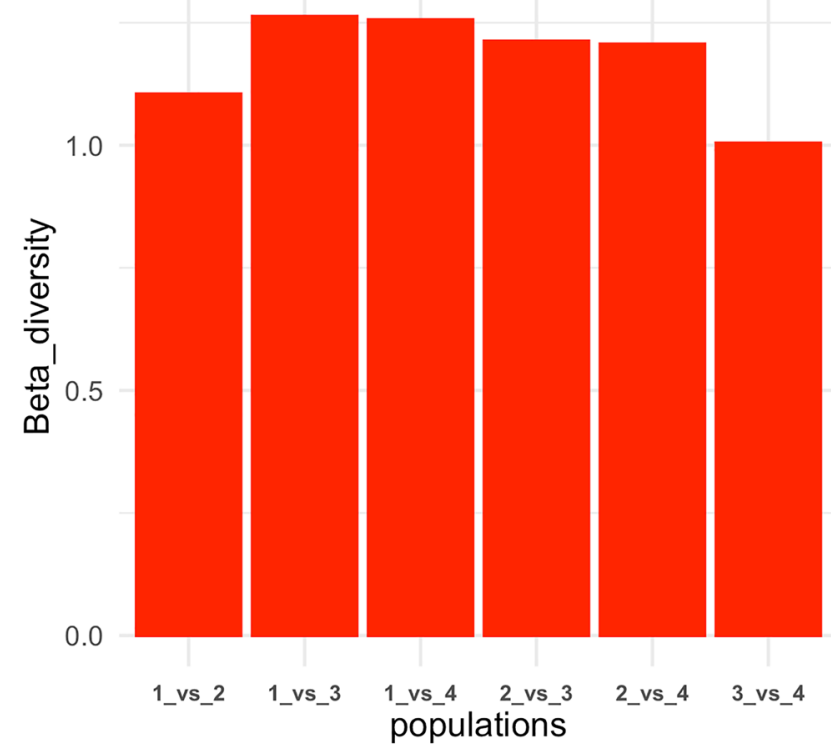

$q=2$

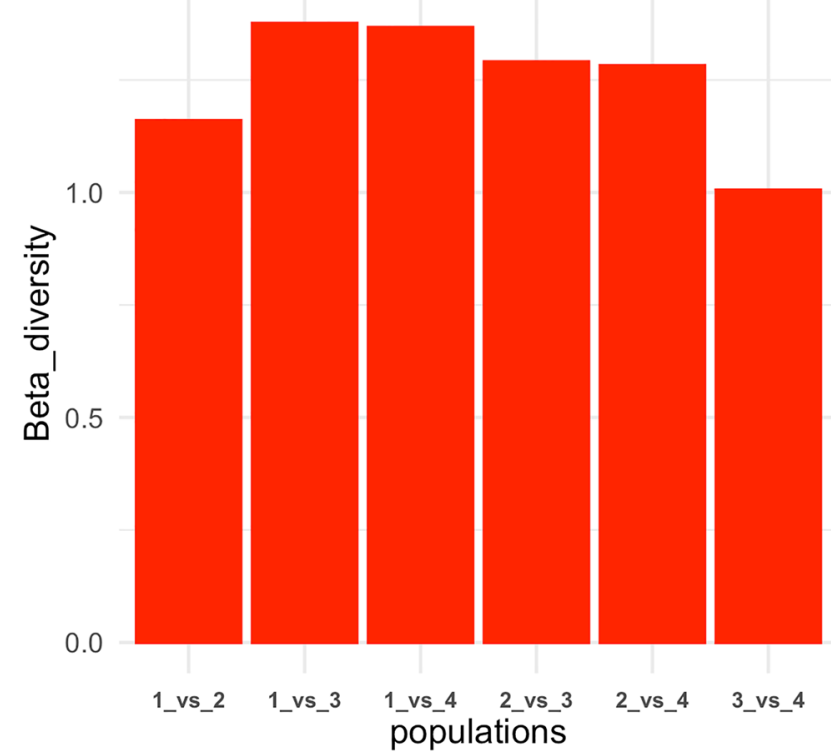

$q=3$
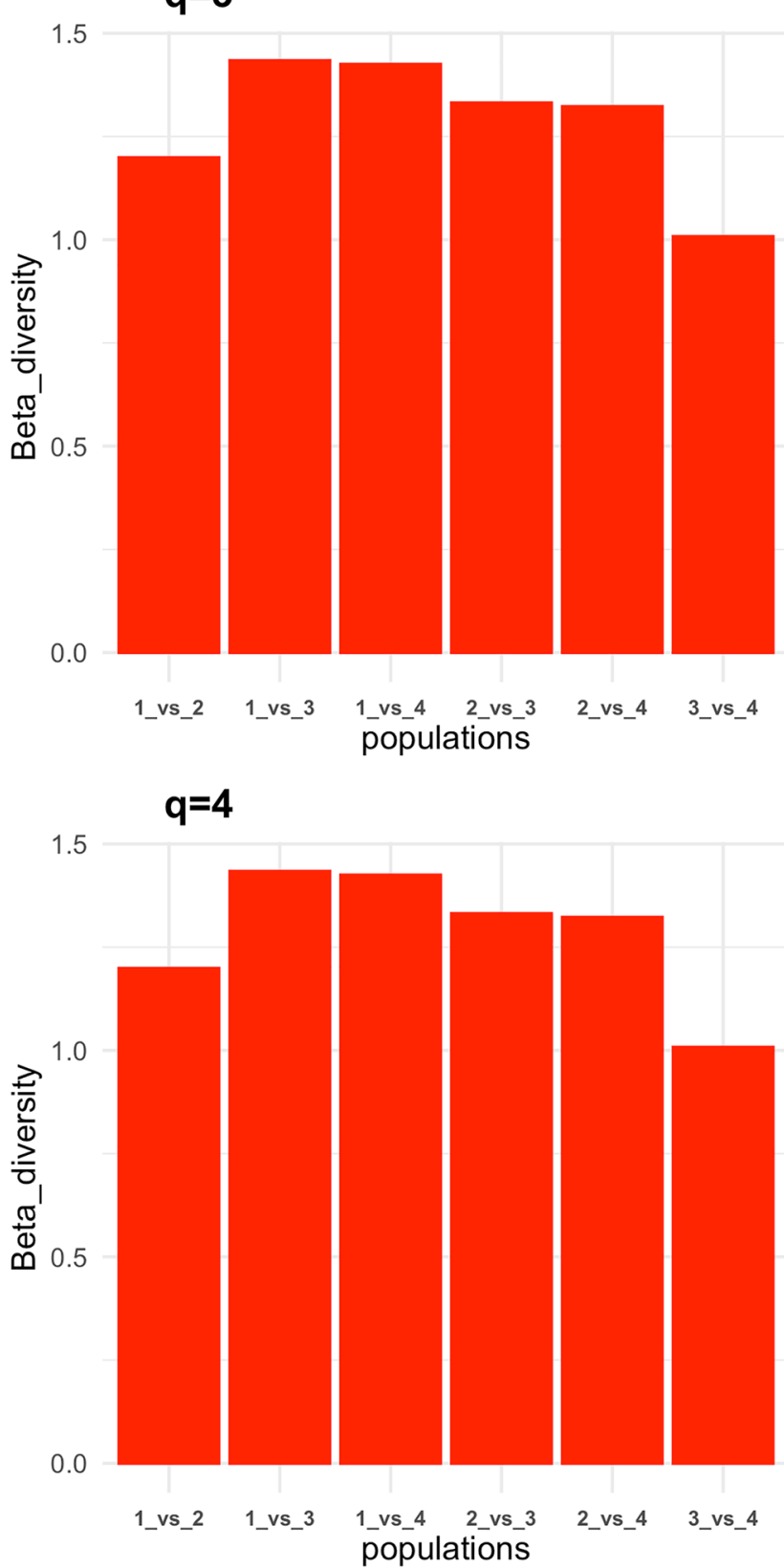

Figure 4. Average autosomal beta diversity at diversity orders $q=1$ to 4 . Populations: (1) Arabian Peninsula dromedary camels; (2) dromedary camels from Iran; (3) Bactrian camels from Iran; and (4) Bactrian camels from Kazakhstan.

The Arabian Peninsula dromedaries appear to be a homogenous population based on PCA, admixture and diversity analyses. Such genetic homogeneity is likely a consequence of high genetic admixture between the subpopulations sampled, possibly driven by the historical use of dromedaries in trading and transportation along the Arabian Peninsula ${ }^{16}$. Another possible factor contributing to gene flow are the breeding practices of camel owners in the Arabian Peninsula, which are generally panmictic, lacking a structured breeding programme such as that typically observed in other livestock ${ }^{17}$. The level of admixture between Arabian Peninsula dromedaries obtained here can explain the observed high relatedness between the dromedary samples in the area. Despite this homogeneity, admixture analyses at $K=2$ and $K=3$ suggest a degree of genetic structure associated with geography, with dromedaries falling into three groups: (1) North (Hurra, Shual, Wodeh and Sofor), Central (Magaheem), West (Hadana and Sahilia); (2) Southwest (Awarik and Awadi); and (3) Southeast (Omani). Such level of genetic structuring has previously been observed on Arabian Peninsula camels using microsatellite genotyping ${ }^{6,7,18}$.

The north and center of the Arabian Peninsula is mainly characterized by plain desert, while the west and southwest of the Arabian Peninsula is characterized by variability in elevation associated with mountains, reaching $3000 \mathrm{~m}$ in altitude ${ }^{19}$, and the coast of the Red Sea. This variable ecology might contribute to genetic differentiation between the dromedary camels populating the different areas. Although the west and southwest camel 

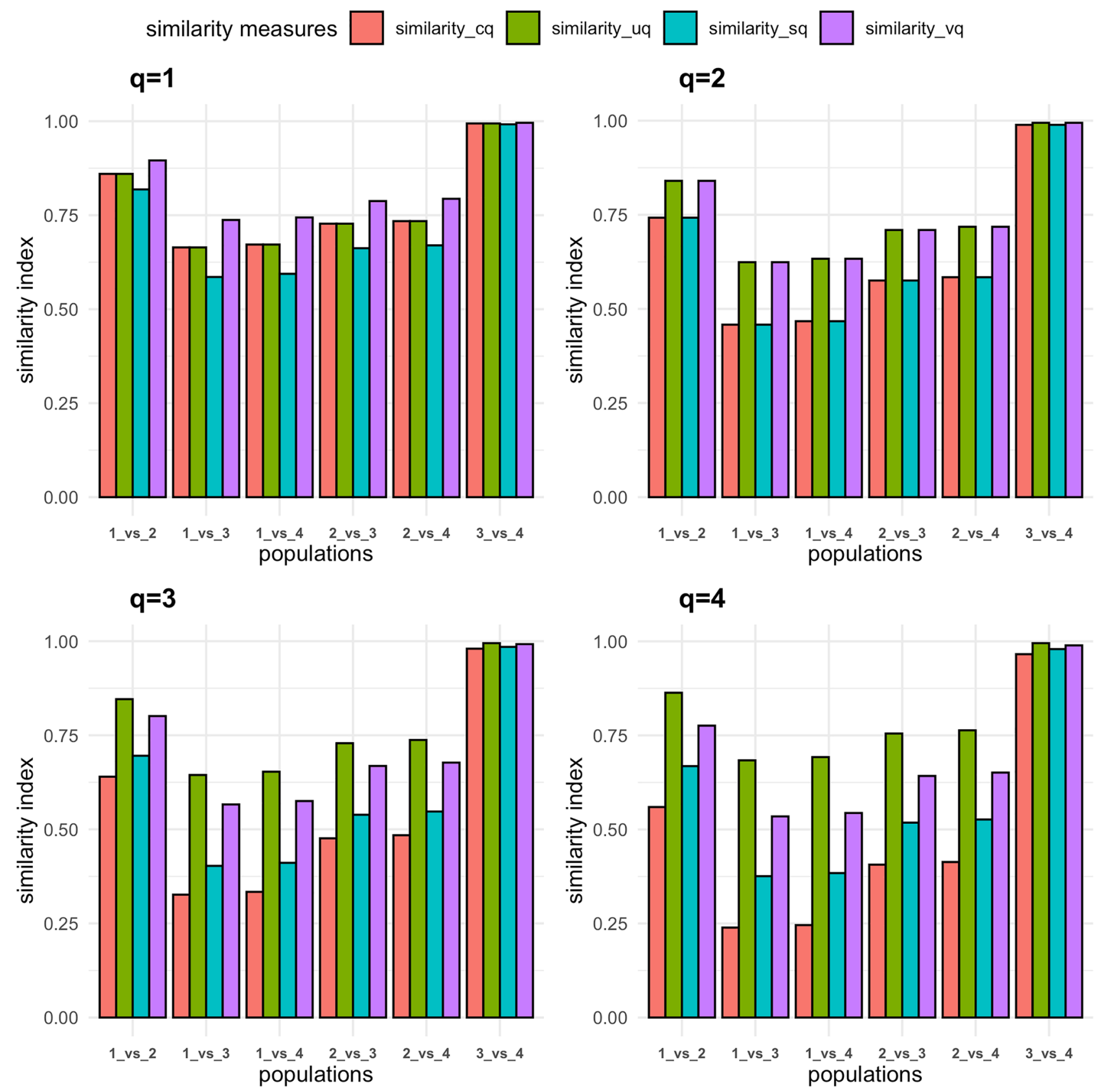

Figure 5. Average autosomal similarity indices at diversity orders $q=1-4$. Populations: (1) Arabian Peninsula dromedary camels; (2) dromedary camels from Iran; (3) Bactrian camels from Iran; and (4) Bactrian camels from Kazakhstan.

populations show a degree of genetic differentiation, their close proximity and likelihood of continuous gene flow may explain the shared genetic ancestry proportion between them. For example, the Awadi camels, which are typically found in the mountains of the Jazan region in the southwest, may also be found along the Red sea coast where Sahlia and Awarik camels are distributed.

The calculated diversity profiles in this study are associated with different advantages benefiting livestock population genetics studies. First, unlike the well-known population-level diversity measures, e.g. heterozygosity, that require large number of samples per population, they are calculated at individual level solving the problem of low number of samples available per population. Second, both of the alpha and beta profiles assess the distribution pattern of the SNPs along each chromosome, or the whole genome, which other diversity measures do not. Third, these profiles can be calculated using other genetic variations, such as insertions, deletions and inversions $^{12}$. 


\begin{tabular}{|l|l|l|}
\hline Population & Geographical location in the Arabian Peninsula & Number of samples \\
\hline Hurra & North & 3 \\
\hline Shual & North & 3 \\
\hline Sofor & North & 2 \\
\hline Wodeh & North & 1 \\
\hline Magaheem & Center & 3 \\
\hline Hadana & West & 2 \\
\hline Sahlia & West & 1 \\
\hline Awadi & Southwest & 2 \\
\hline Awarik & Southwest & 2 \\
\hline Omani & Southeast & 2 \\
\hline
\end{tabular}

Table 2. Dromedary camel populations from Arabian Peninsula included in the study, their geographical distribution and the number of samples in each population.

\section{Conclusion}

We have presented here the first whole-genome sequence analysis of the genetic structure and diversity of Arabian Peninsula dromedary camels. By including dromedary and Bactrian reference populations from outside of the Arabian Peninsula, inter-species and geographic genetic differentiations have been revealed. The Arabian Peninsula camel appear to be a homogenous gene pool with a subtle degree of geographic structure. To validate these findings a larger cohort of camels from populations spanning the whole of the Peninsula is necessary. This study is a first step towards understanding the genetic diversity of dromedaries in the Arabian Peninsula, which is known as their center of domestication.

\section{Materials and methods}

Dromedary samples collection and whole genome DNA extraction. A total of $5 \mathrm{ml}$ blood sampled from the jugular vein was collected from each of 21 dromedary camels using standard techniques approved and in accordance with the relevant guidelines and regulations of the department of veterinary public health and animal husbandry at King Faisal university (Ref: KFU-REC/2018-10-01). All authors compiled with the ARRIVE guidelines $2.0^{20}$. These samples represent dromedary populations from several geographical locations in the Arabian Peninsula (Table 2; Supplementary Table S9). Genomic DNA was extracted using the DNeasy Blood and Tissue kit (Qiagen) according to the manufacturer's protocol.

Whole genome sequence data processing. Genomic DNA was sequenced using paired-end libraries on an Illumina Hiseq 2000 platform at Macrogen in South Korea. Publicly available whole-genome sequence data for four dromedary camels from Iran, six Bactrian camels from Iran, and six Bactrian camels from Kazakhstan (Supplementary Table S9) generated by Ming et al. ${ }^{10}$ was downloaded from NCBI's Sequence Read Archive (https://www.ncbi.nlm.nih.gov/sra).

The fastp software version $0.22 .0^{21}$ was used to trim adapters from the raw sequence reads of the Arabian Peninsula dromedary samples. Raw sequence reads were discarded if: (1) $10 \%$ or more of the read bases were uncertain; (2) $40 \%$ or more of the read bases were low quality (base $\mathrm{Q}_{\text {phred }} \leq 20$ ); (3) reads length was shorter than 15 bases; or (4) read complexity was less than $30 \%$. Bases with quality score $\left(\mathrm{Q}_{\text {phred }}\right)$ less than 20 were also filtered out from the reads. The remaining high-quality reads were mapped against the African dromedary reference genome assembly (CamDro3 $)^{22}$ using the BWA-MEM algorithm implemented in Burrows-Wheeler Aligner (BWA $)^{23}$. Reads were coordinate-sorted using the SortSam option, and PCR-duplicates were marked and excluded using the MarkDuplicates and REMOVE_DUPLICATES = true options in the Picard tools version 1.119 (http://broadinstitue.github.io/picard/index.html). Summary statistics calculated for mapped reads included: the proportion of reference genome coverage via the genomeCoverageBed option implemented in BEDTools software version $2.17^{24}$; mean depth of coverage via the DepthOfCoverage tool implemented in GATK version $3.6^{25}$; and the total number and percentage of mapped reads via the flagstat tool in SAMTools software version $1.19^{26}$.

Variant calling and filtering. Single Nucleotide Polymorphisms (SNPs) were called from the mapped sequence reads using the GATK version 4.1.4.0 HaplotypeCaller tool in GVCF mode. Variants were subsequently combined and genotyped using the GATK CombineGVCFs and GenotypeGVCFs tools in two datasets: dataset 1, which comprised all camel samples; and dataset 2, which comprised only the dromedary camels from the Arabian Peninsula. Quality control filtering criteria were applied on the SNPs of each dataset using the GATK VariantFilteration tool. Parameters included: (1) excluding variants with low quality by depth (QD) (QD $<2) ;(2)$ excluding variants with root mean square of mapping quality for all reads of a site less than $40(\mathrm{MQ}<40.0)$; $(3)$ excluding variants with base quality score less than $30(\mathrm{QUAL}<30)$; $(4)$ excluding variants with high probability of allele-specific strand bias between forward and reverse strand (FS $>60)$; (5) excluding variants with bias in mapping quality between the reads supporting the reference and alternative alleles (MQRankSum $<-12.5)$; and (6) excluding variants with bias in the position of the alternative allele towards the ends of the reads (ReadPosRankSum $<-8$ ). SNPs with a depth of coverage ranging between two reads and three standard deviations from the mean depth of coverage across samples were retained. A total of 14,224,566 and 4,945,503 autosomal SNPs 


\begin{tabular}{|l|r|r|}
\hline \multicolumn{2}{|l|}{} & \multicolumn{2}{l|}{ Datasets } \\
\cline { 2 - 3 } & \multicolumn{1}{|l|}{ Dataset 1 } & Dataset 2 \\
\hline Raw SNPs number & $14,224,566$ & $4,945,503$ \\
\hline Quality control criteria & $2,396,749$ & $1,237,037$ \\
\hline MAF $<5 \%$ & $1,951,013$ & 63,562 \\
\hline Call rate $<95 \%$ & $3,357,791$ & 24 \\
\hline HWE $\left(p-\right.$ value $\left.<1 \times 10^{-6}\right)$ & $6,474,051$ & $3,586,263$ \\
\hline LD $\left(\mathrm{r}^{2}>0.1\right)$ & 44,962 & 58,617 \\
\hline Final number of SNPs retained & &
\end{tabular}

Table 3. Number of autosomal SNPs excluded by filters in dataset 1 (all camel populations) and dataset 2 (Arabian Peninsula dromedary populations).

were retained for dataset 1 and dataset 2, respectively, which are used to calculate SNP diversity and similarity indices.

For the admixture and principal components analyses (PCA), SNPs were further filtered with Plink $1.9^{27}$. Within each dataset, SNPs were excluded if: (1) they had a minor allele frequency $\leq 5 \%$; (2) had a call rate $\leq 95 \%$; or (3) departed significantly from Hardy-Weinberg equilibrium $\left(p\right.$-value $\left.<1 \times 10^{-6}\right)$. SNPs were also filtered to exclude those with a linkage disequilibrium (LD) correlation coefficient $\mathrm{r}^{2}$ value $>0.1$, as in Bahbahani et al. (2019), using Plink's indep-pairwise tool (--indep-pairwise $50 \mathrm{~kb} 10 \mathrm{~kb} 0.1$ ) resulting in 44,962 SNPs in dataset 1 and 58,617 SNPs in dataset 2 (Table 3).

Samples were excluded if: (1) their genotyping call rate was $\leq 95 \%$; or (2) they shared identity-by-state (IBS) $\geq 95 \%$ with another sample, in which case that the lowest genotyping call rate was excluded. A single dromedary camel sample from Iran (SRR5563498) was excluded from dataset 1 due to high IBS with another dromedary sample from Iran (SRR5563500).

Genetic structure and relatedness. PCA was conducted using the prcomp function of R software ${ }^{28}$ on datasets 1 and 2 to determine the genetic relationship between the dromedary and Bactrian samples, and among the dromedary samples from the Arabian Peninsula. Local ancestry admixture analyses were conducted on both datasets using ADMIXTURE $1.23^{29}$, assuming the number of ancestral clusters $(K)$ ranged from 1 to 13 for dataset 1 , and $K$ from 1 to 10 for dataset 2, which reflects the total number of sampled populations in each dataset. A total of 200 bootstrap iterations were performed for each $K$ analysis. The optimal number of clusters was determined as the $K$ value with the lowest cross-validation (CV) error.

Pairwise relatedness tests were conducted on dataset 2 using the --relatedness 2 tool implemented in VCFtools version $1.13^{30}$. This tool implements the Kinship-based INference for Genome-wide association studies (KING) algorithm ${ }^{31}$ to determine relatedness $(\Phi)$. A $\Phi>0.354$ indicates a duplicate sample or monozygotic twin, $0.177<\Phi<0.354$ indicates first-degree relatives, $0.0884<\Phi<0.177$ indicates second-degree relatives, $0.0442<\Phi<0.0884$ indicates third-degree relatives, while $\Phi<0.0442$ indicates the sample-pair to be unrelated ${ }^{31}$.

Individual-level SNP diversity and similarity profiles. The diversity and similarity profiles, i.e. alpha and beta diversities and similarity indices, of autosomal SNPs per gene were calculated for each autosome in datasets 1 and 2 as detailed by Ming et al. ${ }^{10}$. For dataset 1 , four populations were defined: (1) dromedary from Arabian Peninsula; (2) dromedary from Iran; (3) Bactrian from Iran; and (4) Bactrian from Kazakhstan. For dataset 2, the ten Arabian Peninsula dromedary populations were treated as separate populations since the admixture analysis did not reveal any clear clustering. Individual-level alpha diversity profiles were calculated at diversity orders $q=0$ to 4 . The mean alpha diversities were compared between populations using the non-parametric Mann-Whitney $U$ test. For each diversity order, for all pairwise population comparisons in each dataset, we calculated beta diversity in addition to four similarity indices to investigate SNP variation levels: local SNP overlap (Cq); regional SNP overlap (Uq); SNP homogeneity measures (Sq); and SNP turnover complement (Vq).

\section{Data availability}

The Arabian Peninsula dromedary whole genome sequence data analysed during the current study are available in the European Nucleotide Archive (ENA) with the Bioproject accession number (PRJEB47650).

Received: 26 September 2021; Accepted: 15 December 2021

Published online: 07 January 2022

\section{References}

1. Burger, P. A., Ciani, E. \& Faye, B. Old World camels in a modern world-A balancing act between conservation and genetic improvement. Anim. Genet. 50, 598-612. https://doi.org/10.1111/age.12858 (2019).

2. Ali, A., Baby, B. \& Vijayan, R. From desert to medicine: A review of camel genomics and therapeutic products. Front. Genet. 10, 17. https://doi.org/10.3389/fgene.2019.00017 (2019).

3. Al-Swailem, A. M., Al-Busadah, K. A., Shehata, M. M., Al-Anazi, I. O. \& Askari, E. Classification of Saudi Arabian camel (Camelus dromedarius) subtypes based on RAPD technique. J. Food Agric. Environ. 5, 143 (2007). 
4. Wardeh, M. F. Classification of the dromedary camels. J. Camel Sci. 1, 1-7 (2004).

5. Almathen, F., Elbir, H., Bahbahani, H., Mwacharo, J. \& Hanotte, O. Polymorphisms in MC1R and ASIP genes are associated with coat color variation in the Arabian camel. J. Hered. 109, 700-706. https://doi.org/10.1093/jhered/esy024 (2018).

6. AlAskar, H., Alhajeri, B. H., Almathen, F. \& Alhaddad, H. Genetic diversity and population structure of dromedary camel-types. J. Hered. 111, 405-413. https://doi.org/10.1093//hered/esaa016 (2020).

7. Almathen, F. et al. Ancient and modern DNA reveal dynamics of domestication and cross-continental dispersal of the dromedary. Proc. Natl. Acad. Sci. U.S.A. 113, 6707-6712. https://doi.org/10.1073/pnas.1519508113 (2016).

8. Hossam Mahmoud, A., Mohammed Abu-Tarbush, F., Alshaik, M., Aljumaah, R. \& Saleh, A. Genetic diversity and population genetic structure of six dromedary camel (Camelus dromedarius) populations in Saudi Arabia. Saudi J. Biol. Sci. 27, 1384-1389. https://doi.org/10.1016/j.sjbs.2019.11.041 (2020).

9. Bahbahani, H. et al. Genome diversity and signatures of selection for production and performance traits in dromedary camels. Front. Genet. https://doi.org/10.3389/fgene.2019.00893 (2019).

10. Ming, L. et al. Whole-genome sequencing of 128 camels across Asia reveals origin and migration of domestic Bactrian camels. Commun. Biol. 3, 1. https://doi.org/10.1038/s42003-019-0734-6 (2020).

11. Jost, L. Partitioning diversity into independent alpha and beta components. Ecology 88, 2427-2439. https://doi.org/10.1890/06$1736.1(2007)$

12. Ma, Z., Li, L. \& Zhang, Y.-P. Defining individual-level genetic diversity and similarity profiles. Sci. Rep. 10, 5805. https://doi.org/ 10.1038/s41598-020-62362-8 (2020).

13. Chao, A., Chiu, C.-H. \& Jost, L. Unifying species diversity, phylogenetic diversity, functional diversity, and related similarity and differentiation measures through Hill numbers. Annu. Rev. Ecol. Evol. Syst. 45, 297-324. https://doi.org/10.1146/annurev-ecols ys-120213-091540 (2014).

14. Dioli, M. Dromedary (Camelus dromedarius) and Bactrian camel (Camelus bactrianus) crossbreeding husbandry practices in Turkey and Kazakhstan: An in-depth review. Pastoralism 10, 6. https://doi.org/10.1186/s13570-020-0159-3 (2020).

15. Cherifi, Y. A. et al. Weak genetic structure in northern African dromedary camels reflects their unique evolutionary history. PLoS One 12, e0168672. https://doi.org/10.1371/journal.pone.0168672 (2017).

16. Almathen, F. Genetic diversity and demographic history of dromedary camel (Camelus dromedarius). $\mathrm{PhD}$ thesis, Nottingham, (2014).

17. Abri, M. A. A. \& Faye, B. Genetic improvement in dromedary camels: Challenges and opportunities. Front. Genet. 10, 167. https:// doi.org/10.3389/fgene.2019.00167 (2019)

18. Almathen, F. et al. Genetic structure of Arabian Peninsula dromedary camels revealed three geographic groups. Saudi J. Biol. Sci. https://doi.org/10.1016/j.sjbs.2021.11.032 (2021).

19. De Pauw, E. An Agroecological Exploration of the Arabian Peninsula (ICARDA, 2002).

20. Percie du Sert, N. et al. The ARRIVE guidelines 2.0: Updated guidelines for reporting animal research. PLoS Biol. 18, e3000410. https://doi.org/10.1371/journal.pbio.3000410 (2020).

21. Chen, S., Zhou, Y., Chen, Y. \& Gu, J. fastp: An ultra-fast all-in-one FASTQ preprocessor. Bioinformatics 34, i884-i890. https://doi. org/10.1093/bioinformatics/bty560 (2018).

22. Elbers, J. P. et al. Improving Illumina assemblies with Hi-C and long reads: An example with the North African dromedary. Mol. Ecol. Resour. 19, 1015-1026. https://doi.org/10.1111/1755-0998.13020 (2019).

23. Li, H. \& Durbin, R. Fast and accurate long-read alignment with Burrows-Wheeler transform. Bioinformatics 26, 589-595. https:// doi.org/10.1093/bioinformatics/btp698 (2010).

24. Quinlan, A. R. \& Hall, I. M. BEDTools: A flexible suite of utilities for comparing genomic features. Bioinformatics 26, 841-842. https://doi.org/10.1093/bioinformatics/btq033 (2010).

25. McKenna, A. et al. The Genome Analysis Toolkit: A MapReduce framework for analyzing next-generation DNA sequencing data. Genome Res. 20, 1297-1303. https://doi.org/10.1101/gr.107524.110 (2010).

26. Li, H. A statistical framework for SNP calling, mutation discovery, association mapping and population genetical parameter estimation from sequencing data. Bioinformatics 27, 2987-2993. https://doi.org/10.1093/bioinformatics/btr509 (2011).

27. Chang, C. C. et al. Second-generation PLINK: Rising to the challenge of larger and richer datasets. GigaScience 4, 7-7. https://doi. org/10.1186/s13742-015-0047-8 (2015).

28. R: A Language and Environment for Statistical Computing (R Foundation for Statistical Computing, 2019).

29. Alexander, D. H., Novembre, J. \& Lange, K. Fast model-based estimation of ancestry in unrelated individuals. Genome Res. 19, 1655-1664. https://doi.org/10.1101/gr.094052.109 (2009).

30. Danecek, P. et al. The variant call format and VCFtools. Bioinformatics 27, 2156-2158. https://doi.org/10.1093/bioinformatics/ btr330 (2011).

31. Manichaikul, A. et al. Robust relationship inference in genome-wide association studies. Bioinformatics 26, 2867-2873. https:// doi.org/10.1093/bioinformatics/btq559 (2010).

\section{Acknowledgements}

We would like to extend our sincere gratitude to the camel owners who have helped us in sampling dromedary camels. Special thanks to Dr. David Wragg from the Roslin Institute at University of Edinburgh for revising and editing the manuscript.

\section{Author contributions}

H.B. and F.A. conceived and designed the project. F.A. collected the dromedary blood samples from the different locations in the Arabian Peninsula. H.B. performed bioinformatic analysis. H.B. and F.A. wrote the manuscript. All authors have commented upon and agreed on the contents of the manuscript.

\section{Competing interests}

The authors declare no competing interests.

\section{Additional information}

Supplementary Information The online version contains supplementary material available at https://doi.org/ 10.1038/s41598-021-04087-w.

Correspondence and requests for materials should be addressed to H.B.

Reprints and permissions information is available at www.nature.com/reprints. 
Publisher's note Springer Nature remains neutral with regard to jurisdictional claims in published maps and institutional affiliations.

(c) (i) Open Access This article is licensed under a Creative Commons Attribution 4.0 International License, which permits use, sharing, adaptation, distribution and reproduction in any medium or format, as long as you give appropriate credit to the original author(s) and the source, provide a link to the Creative Commons licence, and indicate if changes were made. The images or other third party material in this article are included in the article's Creative Commons licence, unless indicated otherwise in a credit line to the material. If material is not included in the article's Creative Commons licence and your intended use is not permitted by statutory regulation or exceeds the permitted use, you will need to obtain permission directly from the copyright holder. To view a copy of this licence, visit http://creativecommons.org/licenses/by/4.0/.

(c) The Author(s) 2022 